\title{
Nuclear Imaging of a Cardiac Paraganglioma
}

\author{
Nada Almenieir, Shawn Karls, Vilma Derbekyan, and Robert Lisbona \\ Division of Nuclear Medicine, Royal Victoria Hospital, Montreal, Quebec, Canada
}

\begin{abstract}
We report a case of a cardiac paraganglioma in the right atrioventricular groove in which the use of different nuclear medicine studies aided in the diagnosis.
\end{abstract}

Key Words: paraganglioma; cardiac; succinate dehydrogenase/ deficiency

J Nucl Med Technol 2017; 45:247-248

DOI: 10.2967/jnmt.116.182212

\section{$\mathbf{N}$}

uclear imaging is useful for the evaluation of suspected paragangliomas. ${ }^{123} \mathrm{I} /{ }^{131} \mathrm{I}$-metaiodobenzylguanidine (MIBG) scintigraphy is the initial recommended study but is associated with a high false-negative rate, as the sensitivity is $18 \%-50 \%(1)$. Additional nuclear imaging with ${ }^{111}$ In-pentetreotide can be used, with a sensitivity and specificity of $94 \%$ and $75 \%$, respectively (2). Also, studies show that ${ }^{18} \mathrm{~F}$-FDG PET is as specific as ${ }^{123 / 131}$ I-MIBG for detection of the primary tumor and metastases and even more sensitive than ${ }^{123} \mathrm{I}-\mathrm{MIBG}$ and CT/MRI for detection of metastatic disease (3). Mutations in the SDHx genes are associated with paragangliomas. All hereditary $S D H x$ paraganglioma syndromes have an autosomal dominant inheritance pattern with varying penetrance (4).

\section{CASE REPORT}

A 25-y-old woman was referred to the cardiology department because she was experiencing palpitations. An echocardiogram showed a large mass anterior to the right ventricle, measuring $6 \times 5 \mathrm{~cm}$. The mass appeared to be compressing the right ventricle in the right atrioventricular groove. Chest CT (Fig. 1A) and cardiac MRI were performed. CT showed the mass to be heterogeneously hyperenhancing, with a central stellate "scar" centered on the right atrium; the imaging characteristics were suggestive of a cardiac hemangioma. Cardiac paraganglioma was included in the differential diagnosis. Cardiac MRI showed the lesion to be predominantly solid, with several small cystic components, but the scan did not otherwise contribute to the characterization of the lesion.

Received Mar. 13, 2017; revision accepted May 10, 2017.

For correspondence or reprints contact: Nada Almenieir, 1001 Decarie Blvd., Montréal, Québec, Canada H4A 3J1.

E-mail: nada.almenieir@mail.mcgill.ca

Published online Jun. 13, 2017.

COPYRIGHT (c) 2017 by the Society of Nuclear Medicine and Molecular Imaging.
The symptomology was suggestive of a neuroendocrine tumor, with elevated levels of serum chromogranin A, 24-h urinary norepinephrine, dopamine, and normetanephrine. ${ }^{111}$ In-pentetreotide scanning was performed and showed intense increased uptake by the mass (Fig. 1B). To evaluate treatment options, ${ }^{131} \mathrm{I}-\mathrm{MIBG}$ scanning was performed and showed only a mild heterogeneous accumulation of radiotracer in the mass (Fig. 1C). ${ }^{18} \mathrm{~F}-$ FDG PET/CT was performed to rule out metastasis (Fig. 1D). It showed the mass to be intensely hypermetabolic with central necrosis, an $\mathrm{SUV}_{\max }$ of 31, and no metastasis. A multigated acquisition was obtained to evaluate the cardiac dynamics and showed no impairment (left and right ventricular ejection fractions, $64 \%$ and 52\%, respectively).

The mass was surgically resected and the right ventricle repaired with a bovine pericardial patch. Because the presurgical coronary angiogram showed the proximal right coronary artery to be dilated, with a large branch feeding the mass, bypass grafting of the right coronary artery was performed. Pathologic examination confirmed the mass to be a paraganglioma, with a Ki-67 labeling index of $16 \%$. Chromogranin A decreased from 380.7 to $26 \mathrm{ng} / \mathrm{mL}$; $24-\mathrm{h}$ urinary norepinephrine, from 2,249 to $101 \mathrm{nmol} / \mathrm{d}$; dopamine, from 26,096 to $535 \mathrm{nmol} / \mathrm{d}$; and normetanephrine, from 1,266 to $66 \mathrm{nmol} / \mathrm{d}$. Immunohistochemistry showed cytoplasmic loss of expression of succinate dehydrogenase complex, iron-sulfur subunit B. The patient is currently undergoing genetic testing.

\section{DISCUSSION}

In this case, an intracardiac tumor produced a diagnostic dilemma. Although radiographic studies provided structural information, ${ }^{111}$ In-pentetreotide scanning confirmed the clinical suspicion of a neuroendocrine tumor. ${ }^{18} \mathrm{~F}-\mathrm{FDG}$ PET can also be useful and brings the additional value of quantitative SUV. Although the link between the malignant potential of paragangliomas and $\mathrm{Ki}-67$ has not been fully established, in our case the high Ki-67 expression was associated with a high $\mathrm{SUV}_{\max }$.

\section{CONCLUSION}

${ }^{123}$ I-MIBG scans are associated with a high rate of false-negative findings in paragangliomas. ${ }^{111} \mathrm{In}-$ octreotide scintigraphy and ${ }^{18}$ F-FDG PET are useful secondary scans. 


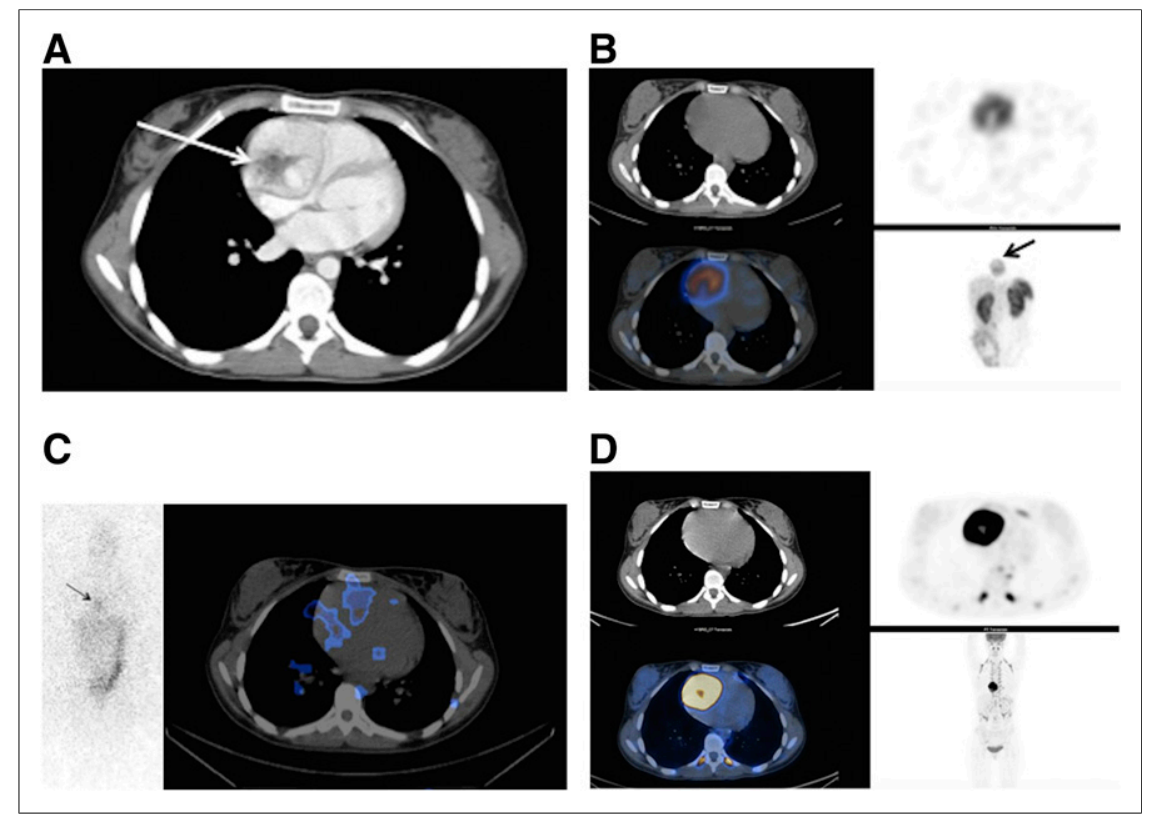

FIGURE 1. (A) Chest CT image showing hyperenhancing mass with hypoenhancing thin rim and central stellate "scar." (B) ${ }^{111}$ In-pentetreotide SPECT/CT images showing focal, intense uptake by mass. (C) 131/-MIBG anterior planar and SPECT/CT images showing only mild heterogeneous radiotracer accumulation in mass. (D) ${ }^{18} \mathrm{~F}-\mathrm{FDG} \mathrm{PET} / \mathrm{CT}$ images showing mass to be intensely hypermetabolic with central necrosis.

A

C

2. Telischi FF, Bustillo A, Whiteman ML, et al. Octreotide scintigraphy for the detection of paragangliomas. Otolaryngol Head Neck Surg. 2000;122: $358-362$.

3. Timmers HJ, Chen CC, Carrasquillo JA, et al. Staging and functional characterization of pheochromocytoma and paraganglioma by ${ }^{18} \mathrm{~F}$-fluorodeoxyglucose $\left({ }^{18}\right.$ F-FDG) positron emission tomography. J Natl Cancer Inst. 2012;104: 700-708.

\section{REFERENCES}

1. Taïeb D, Neumann H, Rubello D, et al. Modern nuclear imaging for paragangliomas: beyond SPECT. J Nucl Med. 2012;53:264-274.

4. Welander J, Söderkvist P, Gimm O. Genetics and clinical characteristics of hereditary pheochromocytomas and paragangliomas. Endocr Relat Cancer. 2011;18: R253-R276. 